\title{
Propiedad y gobernanza: legitimando el derecho a explotar*
}

\author{
Ownership and governance: legitimizing exploitation rights
}

\author{
Historial del artículo \\ Recibido: \\ 14 de abril de 2020 \\ Revisado \\ 15 de junio de 2020 \\ Aceptado: \\ 19 de junio de 2020
}

\section{Álvaro Román ${ }^{\mathrm{a}}$ y Cecilia Campero}

a Centro de Estudios del Desarrollo Regional y Políticas Públicas, Universidad de Los Lagos, Osorno, Chile. Correo electrónico: alvaro.roman@ulagos.cl

${ }^{b}$ School of Public Policy and Global Affairs, and Institute for Resources, Environment and Sustainability, University of British Columbia, Vancouver, Canadá..

\section{Palabras clave}

Explotación, gobernanza, propiedad, recursos naturales

\section{Keywords}

Exploitation, governance, natural resources, ownership

\begin{abstract}
Resumen
Una manera de conocer el estado de la relación entre una sociedad y el espacio es mediante el examen de sus derechos de propiedad. Desde ellos se accede al entramado de relaciones y jerarquías entre grupos sociales, así como al rol que le otorgan al medio en el que se desenvuelven. En este artículo se propone una revisión crítica de dicha relación, una en la que el carácter individual y antrópico de la propiedad no ha sido cuestionado. Con base en entrevistas semiestructuradas y revisión bibliográfica se plantea que las demandas de sectores usualmente perjudicados por esta forma de relación social buscan que se les garantice el derecho a usufructuar recursos bajo paradigmas de mercado. Así, es interesante que los nuevos arreglos institucionales que permiten su integración replican los mismos efectos de desposesión y exclusión. De este modo, las medidas de corrección refuerzan el carácter individual y antrópico de la propiedad que ganan una legitimidad basal. En consecuencia, la asignación de derechos, la definición de grupos prioritarios y las decisiones de carácter político se enfrentan a una acción cada vez más fragmentada, extendiendo la exclusión a grupos cada vez más marginados, además de arriesgar la disponibilidad de los recursos así distribuidos.
\end{abstract}

\section{Abstract}

One way to know the state of the relationship between a society and space is by examining its ownership rights. From them, the network of relationships and hierarchies between social groups, as well as the role they give to the environment in which they operate, can be accessed. This article proposes a critical review of this relationship, one in which the individual and anthropic character of the ownership has not been questioned. Based on semistructured interviews and literature review, it is suggested that the demands of sectors usually affected by this form of social relationship seek to guarantee their right to use resources under market paradigms. Thus, it is interesting that the new institutional arrangements that allow their integration replicates the same effects of dispossession and exclusion. In this way, corrective measures reinforce the individual and anthropic character of ownership, which gains a basal legitimacy. As a result, the allocation of rights, the definition of priority groups and political decisions are faced with increasingly fragmented action, extending exclusion to increasingly marginalized groups, as well as risking the availability of the resources thus distributed. 


\section{Introducción}

Los derechos de propiedad expresan la relación de la sociedad con el espacio (Harm, 2012) y, como parte de ello, las relaciones y jerarquías sociales también se encuentran afectas. La instalación de clases sociales dominantes se fomenta con el binomio perfecto de la propiedad, el derecho a excluir, que trae consigo consecuencias de desposesión y desplazamiento (Blomley, 2015). En términos de Rose (1994), dadas las condiciones de la propiedad y la expectativa de derechos que la conforman, se facilita una "reproducción de jerarquía" (p. 59) que facilita la distancia entre grupos sociales y determina el modo en que estos se desenvuelven en el contexto político. La acumulación de propiedad privada en determinados grupos sociales, en términos urbanos, ha sido expuesta bajo el término landlordism (McCrone \& Elliott, 1989) y, más recientemente, en la literatura esta discusión se ha expandido hacia la naturaleza.

Las tensiones entre la propiedad y el medio natural tuvieron como principal enfoque la concentración y acumulación de los recursos, y su explotación. Sus principales exponentes son Hardin (1968) con la tragedia de los comunes que aborda el agotamiento generado por el ejercicio indiscriminado de los derechos de uso de recursos; y Heller (1998) con la tragedia de los anticomunes que opera como el fracaso de los derechos de exclusión, en la medida que se abre a demasiados participantes la asignación de derechos sobre bienes cada vez más abstractos, cuestión que Andreasson (2006) desarrolla desde la desposesión para definir cómo los derechos de propiedad se expanden de básicos a sofisticados, y cómo la intervención de actores se amplía de la escala local a la global. Esta discusión también se ha trabajado desde la perspectiva de la neoliberalización de la naturaleza con interés en la creación de derechos de propiedad sobre recursos que inicialmente fueron comunes (Bakker, 2008; Castree, 2008; McCarthy \& Prudham, 2004). Con los estudios críticos legales el enfoque de la propiedad, como una institución que se orienta a regular la relación de las personas con objetos, cambió para ser ampliado hacia sus efectos de regulación de las relaciones sociales, donde el poder y el control adoptan un rol en su configuración (Bakker, 2010; Bauer, 2009; Prudham, 2015). En otras palabras, la propiedad descansa sobre una base de legitimidad -respeto, control y sometimiento-que, al mismo tiempo, se ve amenazada por los crecientes conflictos de interés que genera la exclusión.

A partir de la reflexión de Castree (2005) sobre la problemática del término neoliberalización-respecto a que este ha sido utilizado para referirse a una multiplicidad de procesos (privatización, commoditización, desregulación, etc.) al punto de hacer de él un comodín-, Bakker (2008) elaboró una respuesta que incluye el desarrollo de tipologías para distinguir el objetivo y tipo de reforma neoliberal de acuerdo con su relación con tres categorías en el manejo de recursos: instituciones, organizaciones y gobernanza. Al cruzar estas categorías con las estrategias para contrarrestar los efectos de dichas reformas, Bakker (2008) entiende que el principal objetivo de la privatización como institución es la extensión de los derechos de propiedad. Sin embargo, disentimos de su argumentación cuando plantea que las propuestas de alterglobalización son capaces de corregir el carácter individual y antrópico. En este artículo nos proponemos, por el contrario, que las respuestas correctivas de la exclusión generada por la propiedad de recursos naturales están fuertemente permeadas por la estructura actual de esta, despolitizada en el sentido de ser privatizada y con una orientación antrópica y productivista.

El interés por ser acreedor de derechos de propiedad puede ser entendido como un fetiche aspiracional, esto es que está motivado por acceder a las herramientas de las élites: privatización, mercantilización y consumo. Estas herramientas han sido definidas y resguardadas por Estados que también enfrentan una crisis de legitimidad principalmente por la emergencia de nuevas fuerzas (Cerny, 1995; Crouch, 2004), las que apuntan a controlar recursos en disputa. Así, el régimen de gobernanza resultante podría dejar de reflejarse en los derechos de propiedad para ser más bien moldeado por estos.

Planteamos que el derecho a explotar es el destino de una gobernanza de recursos que replica las relaciones sociales reguladas por los derechos de propiedad. Si estos ya contienen inequidades y definiciones arbitrarias de exclusión (Sandel, 2012), es plausible que sean exacerbados en la resolución de controversias más allá de lo estrictamente judicial (Bakker, Kooy, Shofiani \& Martijn, 2008). Si es la propiedad la que genera desposesión y desplazamiento, las nuevas demandas de los sectores usualmente perjudicados por esta forma de relación social apuntan a que se les garantice el derecho a usufructuar recursos bajo paradigmas de mercado, replicándose los mismos efectos de desposesión y exclusión. No se trata solo de acceder a los recursos, sino de aprovecharlos con las herramientas usualmente disponibles para las élites (Smith, 2006). Por medio de su mercantilización, se legitima su explotación. Concluimos que es previsible que la asignación de derechos, la definición de grupos prioritarios o las decisiones de carácter político pueden enfrentarse a una acción cada vez más fragmentada que, especialmente sobre recursos limitados, exagere los 
alcances de la exclusión, además de los efectos sobre la disponibilidad de dichos recursos.

\section{Materiales y métodos}

Para este trabajo se realizó trabajo de campo en varias etapas en Rapa Nui (diciembre de 2017-octubre de 2018) y Chiloé (marzo de 2017-enero de 2019), aplicando entrevistas semiestructuradas, las que fueron sometidas a análisis temáticos, y revisión bibliográfica de artículos que explorasen el rol de la propiedad y los debates locales que dan contexto a los conflictos relacionados con ella. Para ello, se realizaron búsquedas en Google Scholar y Web of Science usando los términos gobernanza, recursos naturales, explotación, propiedad, desposesión y exclusión en revistas y libros. En este artículo exploramos la relación entre propiedad y recursos naturales, y el modo en que dicha relación se ha complejizado a través de una racionalización de la naturaleza como recurso y en diferentes formas de extraer valor de ella. Luego, discutimos el carácter de una sociedad así relacionada con la naturaleza, donde el rol de propietario define no solo una posición jerárquica respecto a otros actores, sino también la capacidad de reproducción social de distintos grupos, enfrentados en una pugna por la exclusividad en el uso de los recursos. Posteriormente, planteamos un debate sobre las medidas de corrección de inequidades en la distribución de la propiedad, la que deviene en la afirmación de la explotación, manteniéndose los problemas basales.

\section{Resultados}

\section{Propiedad, relaciones sociales y recursos naturales}

La expresión acumulación de recursos es frecuentemente utilizada para referirse a la acumulación de propiedad privada en la naturaleza. Un elemento esencial en esta estrategia son los derechos de propiedad, los mismos que lejos de ser estáticos se han expandido hacia nuevas formas de naturaleza (Barnes, 2009). Los recursos comunes o, más reciente, los globales comunes, con frecuencia son utilizados para ejemplificar esta expansión. El debate incluye ejemplos como la atmósfera, con la transacción de bonos de carbono, y el océano, con industrias acuícolas y pesqueras (Mansfield, 2004; McCarthy, 2005).

Una aproximación más reciente en la expansión de derechos de propiedad está en el uso de agua de mar. La desalinización está ganando importancia como tecnología para superar los límites físicos del recurso hídrico, resolver escasez y satisfacer demandas de sectores productivos o, incluso, para abastecer al consumo humano. Sin embargo, hay prácticas y costumbres que están operando ante un silencio de los derechos de propiedad, dando forma a una privatización de este proceso, visible tanto en el abastecimiento de agua potable en California como en la venta del agua producida hacia otros sectores productivos e incluso permitiendo la reconfiguración de las fuentes de agua, como ocurre en la industria minera y agrícola en Chile (Campero \& Harris, 2019; Fragkou \& Budds, 2017; Lee, 2016). Esto muestra cómo el agua se torna un commodity a través de los procesos de desalinización, para permitir nuevas formas de acumulación de capital y reducir presiones para las reformas en el mercado de agua (Fragkou \& Budds, 2017). En términos de Swyngedouw (2013), nos enfrentamos a un nuevo proceso de "acumulación por desalinización” (p. 265).

Mientras algunas aproximaciones enmarcan este paradigma como neoliberalización de la naturaleza (Bakker, 2008; Castree, 2008; McCarthy \& Prudham, 2004), los enfoques críticos legales han enfatizado la propiedad como un instrumento que más allá de reflejar una relación lineal entre persona y objeto, expresa y materializa relaciones sociales (Bakker, 2010; Bauer, 2009; Prudham, 2015). De esta forma, la propiedad implica también la relación entre una persona y la sociedad respecto al objeto, conllevando un reconocimiento de otros respecto del bien (Lindsay, 2015; Rose, 1994). En este sentido, la propiedad (entendida como derecho) y el objeto de propiedad son construcciones sociales. Se puede tener derecho moral sobre la propiedad (la tierra es de quien la trabaja, en el sentido de Locke), pero en tanto exista un desconocimiento de la propiedad por terceros, el derecho moral solo representa un buen argumento para reclamar la propiedad (Lindsay, 2015).

El reconocimiento de propiedad puede ser coercitivo o consentido. De cualquier manera, ninguno de ellos evita cuestionarse si "es legítimo porque las personas lo consienten, o las personas consienten porque es legítimo" (Lindsay, 2015, p.939). Las respuestas al problema de legitimidad se encuentran encerradas en un combate con más propiedad. Esta defensa de la propiedad con más propiedad, según advierte Blomley (2004), parece inevitable en un mundo donde todo es reclamado (emisiones atmosféricas, contaminación hídrica, espacios públicos, etc.) y donde todo está demarcado, o se procura demarcar, por límites. La idea de propiedad como un sitio de lucha es compartida por Egan \& Place (2013), quienes argumentan que además de ser una herramienta para la desposesión es un instrumento de resistencia indígena. Un ejemplo de ello son las reivindicaciones sociales como las de pueblos indígenas mapuche en Chile por sus tierras ancestrales, a través de demandas de identidad (Di Giminiani, 2016) 
y reivindicaciones étnicas, a través del derecho a la consulta plasmado en el Convenio 169 OIT en países como Ecuador y Guatemala (Cisneros, 2016). Se enfrentan así modelos consuetudinarios de propiedad con el régimen de propiedad occidental dominante.

Los regímenes de propiedad han sido caracterizados, principalmente, en cuatro formas: estatal, individual, común y regímenes sin propiedad res nullius (Bromley, 1990). Sin embargo, a partir de los modelos de abastecimiento de agua, Bakker (2008) plantea que estos no son modelos cerrados sino que, por el contrario, adoptan combinaciones. Por ejemplo, el Estado se caracteriza por adoptar un sistema público y/o privado, pero también opera, a través de asociaciones público-privadas, mientras que el mercado (privado o individual, en términos de Bromley, 1990) es netamente privado y la comunidad combina sistemas público-comunes y/o privado. Estos regímenes de propiedad, como menciona Bromley (1990), también pueden mutar en el tiempo. Algunos ejemplos son los procesos de nacionalización que se manifiestan para recuperar la propiedad de los recursos naturales a manos del pueblo. Las experiencias más recientes se identifican en el gobierno de Morales en Bolivia, pero también con Codelco en Chile, Petrobras en Brasil e YPF en Argentina.

Los regímenes de propiedad se distinguen esencialmente a partir de los derechos que los componen (denominados bundle of rights en el sistema anglosajón), mismos que otorgan cualidades en la tenencia y administración, como las figuras de usuario autorizado, solicitante, poseedor y propietario (Schlager \& Ostrom, 1992). Estos regímenes sirven para posicionar a un individuo en el tiempo y espacio vis-à-vis otros, tanto con relación al límite del régimen de propiedad, como dentro del mismo régimen (en caso de haber un grupo o sociedad de propietarios) obteniéndose como resultado la autoridad legítima de actuar y proteger sus intereses con el respaldo del Estado (Bromley, 1990). Como parte de esta multiplicidad de regímenes, también se concretan diferentes metas y resultados que se expresan en condiciones socioeconómicas y en relaciones sociales que definen el acceso y consumo, la administración y la exclusión, entre otros (Rose, 1994).

En el aprovechamiento de recursos, las formas de propiedad han estado influenciadas más por políticas exógenas que endógenas comprendidas como parte de las fuerzas del mercado (Prudham, 2015). Un ejemplo de este proceso es la re-regulación que ocurre a través del Consenso de Washington o en países africanos, con los acuerdos de Bretton Woods (Bakker, 2015). De esta manera se constituyen en un puente entre la propiedad y la legitimación de la explotación, con incidencia en las relaciones de poder y control (Andreasson, 2006; Prudham, 2015). Se trata de una legitimación económica y política, pero que difiere de una legitimación social (Thomson \& Boutilier, 2011).

\section{Hacia una sociedad de propietarios}

De acuerdo con Zurbriggen (2011), el interés por la gobernanza surge como reacción ante un cambio en el modo en que las sociedades occidentales entienden la labor del Poder Ejecutivo. Esta habitualmente ha sido vista como un ejercicio administrativo marcado por la negociación entre agencias estatales y el sector privado, caracterizado principalmente por grandes compañías. En particular, se ha discutido que el rol del Estado ya sea como un garante del bien común o como una estructura puesta al servicio de patrones de crecimiento económico (Apeldoorn, De Graaff \& Overbeek, 2012; Sklair, 2003), está enfrentando una crisis de legitimidad que surge desde sectores que no estaban siendo considerados como partícipes de la toma de decisiones. Zurbriggen (2011) refiere al carácter jerárquico con el que se entiende la toma de decisiones bajo este esquema, y Beck (2008) profundiza en lo que denomina una "trampa territorial" (p. 63) que presupone la existencia del Estado, planteándolo como anterior o por sobre las distintas formas de regulación social.

Si entendemos la gobernanza como el modo en que las sociedades resuelven sus controversias, a través de procedimientos por los cuales se prioriza ciertas decisiones, una lectura más allá de la trampa territorial enriquece la comprensión acerca de las fuerzas y configuración de grupos de interés más diversos, pero, por, sobre todo, menos supeditados de manera formal entre sí. La figura del Estado ha descansado en la noción de obediencia a sus reglas, pero esta se ha visto resquebrajada. El orden dado por la comunión entre Estado y grandes empresas ha dejado profundas crisis sociales y económicas sin resolver, las que han sido abordadas por organizaciones sociales, a través de procesos de negociación que se desprenden de la autoridad estatal para ser llevados a cabo. Cerny (1995) advierte que la mejor prueba de la pérdida de influencia del sector público está dada por la creciente importancia que organismos no gubernamentales tienen en establecer redes en esferas globales. Crouch (2004) elabora una detallada descripción de la pérdida de confianza en los mecanismos gubernamentales, centrada sobre todo en un problema de origen de la relación entre las élites, antes que el enfrentamiento a problemas particularmente distintos a los que se dejaba en manos del Estado en décadas pasadas. 
Los esquemas de gobernanza caracterizados por una multiplicidad de partes interesadas que se enfrentan de manera directa están lejos de ser una respuesta ideal. En ellos las asimetrías entre los participantes del ejercicio de priorización de decisiones no son corregidas, pudiendo incluso exacerbarse. El escenario de desconfianzas que caracteriza Crouch (2004) ha contribuido a problematizar la figura del aparato público, pero no ha dado con un reemplazo, de modo que la regulación de las relaciones sociales es mucho más contingente. Sin embargo, no es sorpresivo que los mejores resultados se concentren en aquellos actores o grupos de interés con capacidades probadas en cuanto a la participación en regímenes de propiedad.

Jessop (2016) recuerda que el Estado todavía es clave en garantizar que los acuerdos entre privados, cualquiera sea la influencia que estos tengan, se respeten valiéndose de las atribuciones punitivas del aparato estatal. Así, podemos identificar dos elementos convergentes: la desconfianza en las capacidades estatales para corregir los efectos de desposesión y la pretensión, especialmente entre grupos sociales que han estado supeditados a las relaciones de propiedad de otros, de tomar parte en una sociedad de propietarios. La legitimidad con que aún cuenta el Estado es utilizada para resolver los más diversos problemas en todos los niveles, a través del reconocimiento del derecho a limitar los usos de los recursos. Luhmann (1993) advierte que la sobrecarga de mecanismos jurídicos, y también del dinero, lleva a una sociedad dividida entre los enardecidos que resultan agraviados -sea por exclusión o por limitación en el uso de tales herramientas- y los que adoptan una posición pasiva ante tales agravios, conformes con los resultados obtenidos. En este sentido es que planteamos que la definición de derechos de propiedad está forjando las relaciones sociales $\mathrm{y}$, en último término, el modo en que las sociedades establecen los caminos para resolver sus controversias.

Reconocemos dos elementos que conducen a una gobernanza definida desde la propiedad. Por un lado, la propiedad forma parte de un relato que asigna no solo una posición jerárquica, sino también la posibilidad de fijar el modo en que la sociedad es organizada (Rose, 1994). En otras palabras, las relaciones sociales que llevan a la desposesión, a través de la exclusión son muy persuasivas. Fontana (1993) refiere a la legitimación de prácticas que, aun siendo perjudiciales, son aceptadas y replicadas por una base social amplia en la medida que estén entre las alternativas menos perjudiciales. Así, si la protección de derechos por parte del Estado no ha sido satisfactoria, tomar el camino de la propiedad supone riesgos de perderla o de no administrarla correctamente, pero al menos permite pasar de ser un sujeto beneficiario a una voz autorizada en los debates públicos.

El segundo elemento es el resultado de un Estado menos mediador y de una mayor variedad de propietarios. La propiedad es lo que está en juego, y esta da unalegitimidad más fuerte que la adopción de un marco ético o la definición de valores defendidos estatalmente. Dado que las controversias son resueltas mediante procedimientos más bien estandarizados que apuntan a tomar menos decisiones y a una menor recurrencia al debate político (cf. Luhmann, 1998), la tendencia es a que haya menos conflictos. Esto tampoco quiere decir que las discrepancias están resueltas de manera automática; al contrario, puede implicar la aceptación de una situación desfavorable a cambio de ser parte de la narrativa de la propiedad. Lukes (2007) enfatiza la dificultad de identificar las relaciones de poder en ausencia de conflictos precisamente porque no es evidente hasta qué punto distintos grupos sociales están consintiendo como resultado de un interés propio o como resultado de la influencia de otros actores a través de la persuasión.

La sofisticación de los derechos de propiedad (Andreasson, 2006) es ejemplificada por reivindicaciones recientes en Chile como las de control en el acceso al territorio en Rapa Nui, la apropiación del borde costero por comunidades lafkenche (Tecklin, 2015) o las de primacía de la población indígena en las formas de consulta ciudadana (Campero \& Barton, 2015). Aylwin (2013) aborda la extensión y sofisticación de derechos poniendo "el consentimiento libre, previo e informado" (p. 68) en el centro del debate acerca de la corrección de las injusticias derivadas de la acumulación; no obstante, las experiencias latinoamericanas, como describe, han sido insatisfactorias. Por ello, una posibilidad es que el régimen de extensión de derechos se acentúe, derivando en la consolidación de una visión extremadamente normativa, desde los derechos, sin que se considere apropiadamente los resultados de estos arreglos institucionales. En tal sentido, la orientación primaria de las organizaciones sociales que plantean reivindicaciones deja de ser el de cuidar el bien común (cf. Bakker, 2008). Así, la sociedad de propietarios no ha introducido correctivos al mercado, sino que este último parece estar reemplazando el modo en que la sociedad establece sus prioridades sobre los recursos.

\section{Consolidando el derecho a explotar}

La manifestación más recurrente del derecho propietario está en el derecho a excluir. Esto se materializa en señaléticas 
con las que se convive a diario, como las que indican propiedad privada, mantenga distancia, o propiedad privada, prohibido pasar (Waldron, 1991). Sin embargo, más allá de la evidente exclusión, la desposesión y los desplazamientos se identifican como efectos de la intensificación de la propiedad, según Rosser (2014) citado por Blomley (2017). Con énfasis en los recursos naturales, Harm (2012) advierte su extensión hacia el derecho a explotar. Estos los definimos como derechos que se otorgan y se crean con el fin de facilitar y complementar las actividades extractivas, condicionado a que esos recursos sean explotados en un contexto de mercado. Como una extensión del derecho a la propiedad, tienen la autoridad legítima de actuar y proteger sus intereses.

La propiedad transforma la relación de la sociedad con la naturaleza y con ello se transforma el manejo de los recursos en sentido operacional (acceso y extracción) y/o en la participación en acciones de decisión colectiva (manejo, exclusión y alienación) (Schlager \& Ostrom, 1992). Las reformas a las regulaciones ambientales, de acuerdo con Bakker (2008), han transformado principalmente tres categorías del manejo de los recursos naturales, específicamente del agua: organizaciones, instituciones y gobernanza. Estas reformas no son necesariamente concomitantes y pueden cubrir una o más categorías a la vez. Los principales tipos de reforma con incidencia en la gobernanza se han llevado adelante a través del mercado, comercialización (reformas en incentivos o sanciones) y devolución o descentralización (Bakker, 2008). No obstante, la asignación de derechos de propiedad desafía al mercado en la extensión de que el control de recursos sea otorgado para la conservación de áreas naturales o protección de modos de vida, a menos que apareje turismo, en tanto actividad orientada a mercantilizar dichas áreas.

En este entender se diferencia entre propiedad para protección económica y propiedad para la eficiencia del mercado (Mansfield, 2004). Un ejemplo del primero son los derechos otorgados como parte de una protección económica hacia un grupo marginalizado, como los Espacios Costeros Marinos de Pueblos Originarios (ECMPO) que procuran resguardar el uso consuetudinario tanto de los recursos como del espacio. Es un proceso irruptivo en el sentido que se lleva a cabo en un territorio caracterizado por su complejidad: la copresencia de actividades acuícolas insertas en cadenas globales que, además, son parte central de la economía chilena (Barton \& Román, 2016; BustosGallardo, 2017) y de comunidades indígenas en una activa afirmación identitaria (Araos et al., 2020). Esta relación no es fluida, sino que tiene espacios de enfrentamiento y resistencia que se forman y conforman por la competencia entre ontologías muchas veces opuestas. La protección de modos de vida, cultura y el tiempo como una dimensión del espacio son con frecuencia recurrentes en las estrategias para combatir la propiedad, especialmente a través de la incorporación de nuevos actores en una gobernanza basada en el control de los recursos (Anbleyth-Evans et al., 2020). Sin embargo, también son utilizadas con trasfondos que buscan la eficiencia del mercado (Tecklin, 2015). Se trata de intentos por regular los efectos adversos de la propiedad con más propiedad no para la conservación, sino para tener la titularidad de la explotación y ejercer los derechos que lo componen.

El segundo tipo de propiedad lo encontramos en el caso del pueblo rapa nui, que en el año 2016 logró un acuerdo con el Estado chileno para administrar el Parque Nacional Rapa Nui, ya existente, pero dirigido por un organismo centralizado. Este parque, una reserva natural y arqueológica, es el principal atractivo turístico de la isla Rapa Nui y el motor de su actividad turística. Al igual que lo propuesto por Anbleyth-Evans et al. (2020), en este caso también se ha generado una exclusión de los habitantes del territorio y sus usuarios más directos. Particularmente, se produjo una discrepancia con el fuerte sesgo arqueológico en la administración del parque, por sobre la valoración de su cultura viva y, sobre todo, de la fragilidad de la isla (Figueroa \& Rotarou, 2016; Ramírez, 2004). Desde la administración del parque, la comunidad indígena ha establecido nuevas pautas de exclusión de actividades y usos, que involucra, entre otras cosas, restricciones al asentamiento de chilenos en la isla (Ley 21.070), pero también supone un conflicto abierto sobre la legitimidad de los directivos de la nueva administración, los mecanismos de control interno y la distribución de los beneficios reportados por esta concesión estatal.

Estos son casos paradigmáticos porque atañen a comunidades indígenas que en alguna medida han optado por mecanismos colectivos para regular el uso de bienes comunes. Sin embargo, en la actualidad están adoptando mecanismos estatales de privatización. Como consecuencia, una vez definida la propiedad, el desenvolvimiento de las relaciones sociales queda legitimado por reglas de mercado. El derecho a explotar recursos naturales no garantiza la continuidad de un modo de vida ni evita el agotamiento de tales recursos, y mucho menos garantiza la resolución de conflictos. En lugar de ello, puede legitimar un dinamismo económico precisamente a costa de modos de vida y recursos, como ha ocurrido en Copiapó, donde el acceso a derechos de agua no ha evitado su sobreexplotación, llevando a los agricultores a abandonar su actividad y sumarse al mercado inmobiliario (Astudillo, 2014). La propiedad del suelo 
(o el derecho a excluir a otros de ese suelo) tampoco ha satisfecho las expectativas de vida de quienes la detentan (Hidalgo, Urbina, Alvarado \& Paulsen, 2017). Son casos en que la propiedad es fetichizada, convirtiéndose en un objetivo que, aunque adquiera rasgos de reivindicación social, ofrece soluciones de carácter individual, y tratándose de individuos que parten en una situación de desventaja -sea porque no cuentan con alternativas reales para ejercer su derecho a explotar o porque ingresan a un sistema de relaciones con códigos que no han sido internalizadosenfrentan un escenario marcadamente asimétrico.

La reivindicación del derecho a explotar conlleva otra consecuencia, que podemos entender como una suerte de gentrificación institucional. En la definición de LópezMorales (2013), la gentrificación es un proceso de expulsión de población de bajos recursos por habitantes que no necesariamente tienen un poder adquisitivo elevado, sino que basta con que se sitúen apenas por sobre la capacidad de consumo de los antiguos residentes. En el derecho a explotar encontramos una analogía similar en el sentido que la consecuencia de desposesión que se genera por la asignación de derechos de propiedad afecta en primer lugar a grupos que se encuentran más fragmentados o con menores herramientas de reconocimiento de derechos. En tal sentido, la reivindicación del derecho a explotar resulta una estrategia irreflexiva porque lejos de resolver la desposesión, la lleva a niveles de creciente especificidad. Por otro lado, mientras que el capital es móvil y las decisiones de inversión tienen cierto margen de movilidad, comunidades, modos de vida y recursos naturales son frágiles y vulnerables a decisiones orientadas desde los derechos de explotación. En tal sentido, el reemplazo de un régimen de gobernanza por un sistema propietario expone a que la racionalidad económica sea no solo legitimada, sino también una aspiración precisamente por quienes han cuestionado sus impactos.

\section{Conclusiones}

El derecho a explotar es la expresión del desgaste de la política como mecanismo de toma de decisiones. $\mathrm{Su}$ deslegitimación, las narrativas de menor eficiencia del aparato estatal respecto a la gestión del sector privado y la pretensión de ser parte de la sociedad de propietarios han contribuido a que el reconocimiento social pase necesariamente por la propiedad. Sin embargo, también emerge como respuesta a las insuficiencias del mercado en términos de resguardar el bien común. El hecho de carecer de propiedad supone el riesgo de ser excluido, de ahí que la extensión de derechos se constituya en la estrategia para tomar un lugar, para ganar reconocimiento, en las relaciones sociales constituidas a partir de la legitimación del propietario. Bakker et al. (2008) señalan que en un contexto de desregulación se tiende a generar los peores resultados para la población de menores ingresos. Antes que emparentar el nivel de ingresos con las reivindicaciones de propiedad, se enfatiza que ser portador de derechos ha sido visto como una forma eficiente de garantizar estándares mínimos (Rose, 1994). Así, no resulta extraño que las reivindicaciones por el derecho a explotar surjan desde sectores habitualmente agraviados por los efectos de desposesión derivados de la propiedad.

No obstante, el derecho a explotar no parece estar mitigando dichos efectos, sino que los extiende a cada vez más recursos y a escalas que confrontan a grupos sociales cada vez más específicos. La demanda por propiedad sugiere que la gobernanza ya no cumple un rol en la mediación de controversias, y el énfasis en el ejercicio de estos derechos bajo criterios de mercado plantea desafíos en términos del uso de recursos y de su asignación a grupos prioritarios. La noción de gentrificación institucional nos permite examinar que las normas generadas para satisfacer las reivindicaciones de propiedad conducen a fenómenos de exclusión cada vez más fragmentados. La extensión de la propiedad a recursos cada vez más abstractos implica, además, que el ejercicio de exclusión se amplíe (Andreasson, 2006), afectando a prácticas y modos de vida. Para defenderlos se recurrirá al reconocimiento de nuevos propietarios, razón por la que decimos que el derecho a explotar es una estrategia irreflexiva.

La legitimación como estrategia para conseguir y resistir el derecho a explotar no solo desafía la capacidad del Estado como protector de intereses, sino que también expande los regímenes de propiedad en sus distintas modalidades, sin lograr evadir los efectos perversos que la conforman. Como resultado, es común que los individuos y/o grupos sociales dejen de cuestionar el tipo de propiedad deseado, para enfatizar más en los resultados que esperan alcanzar a partir de la propiedad como institución (Lindsay, 2015). En tal sentido, la reivindicación del derecho a explotar, lejos de resolver los efectos de la propiedad, conduce a esferas de mayor especificidad que nada tienen que ofrecer en términos de garantizar modos de vida, evitar el agotamiento de recursos y, más aun, evitar la exclusión de actividades y usos de otros grupos sociales.

\section{Referencias}

Anbleyth-Evans, J., Araos, F., Ther, F., Segovia, R., Häussermann, V. \& Aguirre-Munoz, C. (2020). Toward marine democracy in Chile: examining 
aquaculture ecological impacts through common property local ecological knowledge. Marine Policy, 113. https://doi.org/10.1016/j.marpol.2019.103690

Andreasson, S. (2006). Stand and deliver: private property and the politics of global dispossession. Political Studies, 54(1), 3-22. https://doi. org/10.1111/j.1467-9248.2006.00563.x

Apeldoorn, B. Van, De Graaff, N. \& Overbeek, H. (2012). The reconfiguration of the global State-capital nexus. Globalizations, 9(4), 471-486. https://doi.org/10.108 0/14747731.2012.699915

Araos, F., Catalán, E., Álvarez, R., Núñez, D., Brañas, F. \& Riquelme, W. (2020). Espacios Costeros Marinos para Pueblos Originarios: usos consuetudinarios y conservación marina. Anuário Antropológico, I, 4768. https://doi.org/10.4000/aa.4933

Astudillo, F (2014) ¿Acumulación por desposesión hídrica? Crecimiento inmobiliario, neoliberalismo minero y mercantilización del agua en Copiapó, Chile. Ecología Política, 47, 62-66. Recuperado de https://www.ecologiapolitica.info/?p=1626

Aylwin, J. (2013). Mercados y derechos globales: implicancias para los pueblos indígenas de América Latina y Canadá. Revista de Derecho, 24(2), 67-91. http://dx.doi.org/10.4067/S071809502013000200003

Bakker, K. (2008). The "Commons" versus the "Commodity": alter-globalization, anti-privatization, and the human right to water in the Global South. En Mansfield, B. (Ed.), Privatization: property and the remaking of nature-society relations (pp. 38-63). Oxford: Blackwell Publishing.

Bakker, K. (2010). The limits of 'neoliberal natures': debating green neoliberalism. Progress in Human Geography, 34(6), 715-735. https://doi. org/10.1177/0309132510376849

Bakker, K. (2015). Neoliberalization of nature. En Perreault, T., Bridge, G. \& McCarthy, J. (Eds.), The Routledge Handbook of Political Ecology (pp. 446456). New York: Routledge.

Bakker, K., Kooy, M., Shofiani, N. E. \& Martijn, E.-J. (2008). Governance failure: rethinking the institutional dimensions of urban water supply to poor households. World Development, 36(10), 1891-1915. https://doi.org/10.1016/j.worlddev.2007.09.015

Barnes, R. (2009). Property rights and natural resources. Oxford; Portland: Hart Publishing.

Barton, J.R. \& Román, Á. (2016). Sustainable development? Salmon aquaculture and late modernity in the archipelago of Chiloé, Chile. Island Studies Journal, 11(2), 651-672. Recuperado de https://www. islandstudies.ca/sites/islandstudies.ca/files/ISJ-11-2MS369-Barton+Roman.pdf

Bauer, C. (2009). Dams and markets: rivers and electric power in Chile. Natural Resources Journal, 49(3-4), 583-651. Recuperado de https://arizona.pure.elsevier. $\mathrm{com} / \mathrm{en} /$ publications/dams-and-markets-rivers-andelectric-power-in-chile

Beck, U. (2008). ¿Qué es la globalización? Falacias del globalismo, respuestas a la globalización. Barcelona: Paidós.

Blomley, N. (2004). The boundaries of property: lessons from Beatrix Potter. The Canadian Geographer, 48(2), 91-100. https://doi.org/10.1111/j.00083658.2004.00049.x

Blomley, N. (2015). The territory of property. Progress in Human Geography, 40(5), 593-609. https://doi. org/10.1177/0309132515596380

Blomley, N. (2017). Land use, planning, and the "difficult character of property". Planning Theory \& Practice, 18(3), 351-364. https://doi.org/10.1080/146 49357.2016.1179336

Bromley, D. (1990, septiembre). The commons, property and common property regimes [conferencia]. Trabajo presentado en la Conferencia "Designing Sustainability on the Commons". Durham, EE.UU. Recuperado de https://dlc.dlib.indiana.edu/dlc/ handle/10535/443

Bustos-Gallardo, B. (2017). The post 2008 Chilean salmon industry: an example of an enclave economy. The Geographical Journal, 183(2), 152-163. https:// doi.org/10.1111/geoj.12204

Campero, C. \& Barton, J. R. (2015). 'You have to be with God and the Devil': linking Bolivia's extractive industries and local development through social 
licences. Bulletin of Latin American Research, 34(2), 167-183. https://doi.org/10.1111/blar.12260

Campero, C. \& Harris, L. M. (2019). The legal geographies of water claims: seawater desalination in mining regions in Chile. Water, 11(5), 886. https:// doi.org/10.3390/w11050886

Castree, N. (2005). The epistemology of particulars: Human Geography, case studies, and "context". Geoforum, 36(5), 541-666. http://dx.doi. org/10.1016/j.geoforum.2005.08.001

Castree, N. (2008). Neoliberalising nature: the logics of deregulation and reregulation. Environment and Planning A, 40(1), 131-152. https://doi.org/10.1068/a3999

Cerny, P. G. (1995). Globalization and the changing logic of collective action. International Organization, 49(4), 595-625. https://doi.org/10.1017/ S0020818300028459

Cisneros, P. (Ed.) (2016). Política minera y sociedad civil en América Latina. Ecuador: Editorial IAEN. Recuperado de https://editorial.iaen.edu.ec/wpcontent/uploads/2017/02/POLÍTICA-MINERAY-SOCIEDAD-CIVIL-EN-AMÉRICA-LATINAfinal-2.pdf

Crouch, C. (2004). Post-democracy. Cambridge: Polity Press.

Di Giminiani, P. (2016). Being from the land: memory, self and the power of place in Indigenous Southern Chile. Ethnos, 81(5), 888-912. https://doi.org/10.108 0/00141844.2015.1028566

Egan, B. \& Place, J. (2013). Minding the gaps: property, geography, and Indigenous peoples in Canada. Geoforum, 44, 129-138. https://doi.org/10.1016/j. geoforum.2012.10.003

Figueroa, E. \& Rotarou, E. S. (2016). Tourism as the development driver of Easter Island: The key role of resident perceptions. Island Studies Journal, 11(1), 245-264. Recuperado de http://www.islandstudies. $\mathrm{ca} / \mathrm{sites} /$ islandstudies.ca/files/ISJ-11-1-O-FigueroaRotarou.pdf

Fontana, B. (1993). Hegemony and power: on the relation between Gramsci and Machiavelli. Minneapolis: University of Minnesota Press.
Fragkou, M. \& Budds, J. (2017, septiembre). Desalination and the disarticulation of the hydrosocial cycle: stabilising the neoliberal model in Chile [conferencia]. Trabajo presentado en la RGS-IBG Annual International Conference. Londres, Reino Unido. http://conference.rgs.org/AC2017/360

Hardin, G. (1968). The tragedy of the commons. Science, 162(3859), 1243-1248. http://doi.org/10.1126/ science.162.3859.1243

Harm, M. (2012). Mining sacred space: law's enactment of competing ontologies in the American West. Environment and Planning A, 44(6), 1443-1458. https://doi.org/10.1068/a44579

Heller, M. A. (1998). The tragedy of the anticommons: property in the transition from Marx to markets. Harvard Law Review, 111(3), 621-688. Recuperado de https://repository.law.umich.edu/articles/609/

Hidalgo, R., Urbina, P., Alvarado, V. \& Paulsen, A. (2017). Desplazados y iolvidados?: contradicciones respecto de la satisfacción residencial en Bajos de Mena, Puente Alto, Santiago de Chile. Revista INVI, 32(89), 85-110. Recuperado de http://revistainvi. uchile.cl/index.php/INVI/article/view/1073

Jessop, B. (2016). Territory, politics, governance and multispatial metagovernance. Territory, Politics, Governance, 4(1), 8-32. https://doi.org/10.1080/216 22671.2015.1123173

Lee, M. (2016). Privatization of water desalination: the need to balance governmental and corporate control in California. The Global Business Law Review, 5(1), 23-49. Recuperado de https://engagedscholarship. csuohio.edu/gblr/vol5/iss1/3/

Lindsay, P. (2015). Ownership by agreement. Political Studies, 63(4), 935-950. https://doi.org/10.1111/14679248.12138

López-Morales, E. (2013). Gentrificación en Chile: aportes conceptuales y evidencias para una discusión necesaria. Revista de Geografia Norte Grande, 56, 31-52. http://dx.doi.org/10.4067/S071834022013000300003

Luhmann, N. (1993). Teoría política en el Estado de Bienestar. Madrid: Alianza Editorial. 
Luhmann, N. (1998). Complejidad y modernidad: de la unidad a la diferencia. Madrid: Trotta.

Lukes, S. (2007). El poder: un enfoque radical. Madrid: Siglo XXI.

Mansfield, B. (2004). Neoliberalism in the oceans: "rationalization," property rights, and the commons question. Geoforum, 35(3), 313-326. https://doi. org/10.1016/j.geoforum.2003.05.002

McCarthy, J. (2005). Commons as counterhegemonic projects. Capitalism Nature Socialism, 16(1), 9-24. https://doi.org/10.1080/1045575052000335348

McCarthy, J. \& Prudham, S. (2004). Neoliberal nature and the nature of neoliberalism. Geoforum, 35(3), 275283. https://doi.org/10.1016/j.geoforum.2003.07.003

McCrone, D. \& Elliott, B. (1989). Property and power in a city: the sociological significance of landlordism. London: The Macmillan Press. Recuperado de https:// www.palgrave.com/gp/book/9781349169252

Prudham, S. (2015). Property and commodification. En Perreault, T., Bridge, G. \& McCarthy, J. (Eds.), The Routledge Handbook of Political Ecology (pp. 430445). New York: Routledge.

Ramírez, J. M. (2004). Manejo del recurso arqueológico en Rapa Nui: teoría y realidad. Chungará, 36(1), 489-497. http://dx.doi.org/10.4067/S071773562004000300049

Rose, C. (1994). Property and persuasion: essays on the history, theory, and rhetoric of ownership. Boulder: Westview Press. Recuperado de https://www.law. yale.edu/sites/default/files/documents/pdf/Faculty/ propertypersuasion.pdf

Sandel, M. J. (2012). What money can't buy: the moral limits of markets. New York: Farrar, Strauss, and Giroux.

Schlager, E. \& Ostrom, E. (1992). Property-rights regimes and natural resources: a conceptual analysis. Land Economics, 68(3), 249-262. http://doi. org/10.2307/3146375
Sklair, L. (2003). Sociología del sistema global: el impacto socioeconómico y político de las corporaciones transnacionales. Barcelona: Editorial Gedisa.

Smith, N. (2006). Foreword. En: Heynen, N., Kaika, M. \& Swyngedouw, E. (Eds.), In the nature of cities: urban political ecology and the politics of urban metabolism, cap. xi-xv. Nueva York: Routledge.

Swyngedouw, E. (2013). Into the sea: desalination as hydro-social fix in Spain. Annals of the Association of American Geographers, 103(2), 261-270. https:// doi.org/10.1080/00045608.2013.754688

Tecklin, D. (2015). La apropiación del litoral de Chile: la ecología política de los derechos privados en torno al mayor recurso público del país. En Bustos, B., Prieto, M. \& Barton, J. R. (Eds.), Ecología política en Chile: naturaleza, propiedad, conocimiento y poder (pp. 121-141). Santiago de Chile: Editorial Universitaria.

Thomson, I. \& Boutilier, R. (2011). Modelling and measuring the social license to operate: fruits of a dialogue between theory and practice. Recuperado de https://socialicense.com/publications/Modelling $\% 20$ and $\% 20$ Measuring $\% 20$ the $\% 20$ SLO.pdf

Waldron, J. (1991). Homelessness and the issue of freedom. UCLA Law Rev, 39, 295-324. Recuperado de https://constcourt.ge/files/2/Journal2019.1/ Jeremy-Waldron-2019.1eng.pdf

Zurbriggen, C. (2011). Gobernanza: una mirada desde América Latina. Perfiles Latinoamericanos, 19(38), 39-64. Recuperado de https://perfilesla.flacso.edu. mx/index.php/perfilesla/article/view/124

\section{Agradecimientos}

Los autores agradecen el apoyo a través de los proyectos ANID/FONDECYT/3170740 y ANID/Becas Chile/74180023. 